\title{
ZEROS OF FUNCTIONS OF REGULAR GROWTH ON A LINE
}

\author{
W. D. BOUWSMA ${ }^{1}$
}

1. Introduction. Levinson [2] and others have worked on the distribution of zeros of functions of exponential type which satisfy conditions similar to boundedness on the real axis. For such functions Levinson showed that the expression $\sum r_{k}^{-1} \sin \theta_{k}$ is bounded, where the zeros of the function are $z_{k}=r_{k} e^{i \theta_{k}}$. It is the purpose of this paper to obtain results concerning the distribution of zeros of functions of exponential type and regular exponential growth on the real axis.

We consider a function $f(z)$ of exponential type having the property that $K=\lim _{x \rightarrow \infty}^{*} x^{-1} \log |f(x) f(-x)|$ exists, where the asterisk indicates that one may discard certain intervals about the zeros of $f(z)$ in the evaluation of the limit, and these intervals can be taken so small as to form a set of logarithmic measure zero. The main result is that for such a function, if the zeros of $f(z)$ in the upper half-plane are denoted by $z_{k}=r_{k} e^{i \theta_{k}}$, then

$$
\lim _{R \rightarrow \infty}(\log R)^{-1} \sum_{r_{k} \leq R} r_{k}^{-1} \sin \theta_{k}=K / 2 \pi
$$

Moreover, if $f(z)$ is of exponential type, and if the limit superior of the left-hand side of (1) is $L$, it is shown that $\lim \sup _{R \rightarrow \infty} R^{-1} n(R) \geqq L$. Thus regular exponential growth on the real axis does yield information on the distribution of zeros of $f(z)$, although this information is less precise than that obtained from conditions such as boundedness on the real axis.

2. Zeros and an integral description of growth. Since our interest is in the asymptotic behavior of $f(z)$, there is no loss of generality in assuming that $f(0)=1$. We denote the zeros of $f(z)$ in the upper halfplane by $z_{k}=r_{k} e^{i \theta_{k}}$.

THEOREM 1. If $f(z)$ is of exponential type, then

$$
\lim _{R \rightarrow \infty}(\log R)^{-1} \sum_{r_{k} \leq R} r_{k}^{-1} \sin \theta_{k}=\lim _{R \rightarrow \infty}(\log R)^{-1} \int_{1}^{R} x^{-2} \log |f(x) f(-x)| d x
$$

\section{if either limit exists.}

Received by the editors July $5,1963$.

1 This paper formed part of a doctoral dissertation at the University of Michigan. The author is indebted to Professor Wilfred Kaplan for his helpful guidance. 
Proof. Carleman's formula asserts that

(2) $\begin{aligned} & \sum_{r_{k} \leq R}\left(r_{k}^{-1}-R^{-2} r_{k}\right) \sin \theta_{k}=(\pi R)^{-1} \int_{0}^{\pi} \log \left|f\left(R e^{i \theta}\right)\right| \sin \theta d \theta \\ &+(2 \pi)^{-1} \int_{0}^{R}\left(x^{-2}-R^{-2}\right) \log |f(x) f(-x)| d x+A,\end{aligned}$

where $A$ is a constant. The theorem will be proved by making estimates on various terms of (2).

We first show that as $R \rightarrow \infty$,

$$
B(R)=(\pi R)^{-1} \int_{0}^{\pi} \log \left|f\left(R e^{i \theta}\right)\right| \sin \theta d \theta=O(1) .
$$

It is clear that $B(R) \leqq O(1)$. Now if we write

$$
\log \left|f\left(R e^{i \theta}\right)\right|=\log ^{+}\left|f\left(R e^{i \theta}\right)\right|+\log ^{-}\left|f\left(R e^{i \theta}\right)\right|,
$$

and observe from Jensen's formula that

$$
\int_{0}^{2 \pi} \log \left|f\left(R e^{i \theta}\right)\right| d \theta \geqq 0,
$$

a simple computation shows that $B(R) \geqq O(1)$, and (3) follows.

We next show that if $\left\{z_{k}\right\}=\left\{r_{k} e^{i \theta_{k}}\right\}$ is any countable set of points in the upper half-plane with no finite limit point,

(4) $\lim _{R \rightarrow \infty}(\log R)^{-1} \sum_{r_{k} \leq R}\left(r_{k}^{-1}-R^{-2} r_{k}\right) \sin \theta_{k}=\lim _{R \rightarrow \infty}(\log R)^{-1} \sum_{r_{k} \leq R} r_{k}^{-1} \sin \theta_{k}$, if either limit exists.

Suppose the limit of the left-hand side of (4) exists and is denoted by $L$. Writing the right-hand side of (4) as $\lim _{R \rightarrow \infty} C(R)$, it is immediate that $\lim \inf _{R \rightarrow \infty} C(R) \geqq L$. On the other hand, let $n$ be any positive integer and observe that

$$
\begin{aligned}
\sum_{r_{k} \leq R}\left(r_{k}^{-1}-R^{-2} r_{k}\right) \sin \theta_{k} & \geqq \sum_{r_{k} \leq R / n}\left(r_{k}^{-1}-R^{-2} r_{k}\right) \sin \theta_{k} \\
& \geqq n^{-2}\left(n^{2}-1\right) \sum_{r_{k} \leq R / n} r_{k}^{-1} \sin \theta_{k} .
\end{aligned}
$$

Now divide (5) by $\log (R / n)$ and let $R \rightarrow \infty$. We see that

$$
\frac{n^{2}-1}{n^{2}} \limsup _{R \rightarrow \infty} \sum_{r_{k} \leq R / n} \frac{r_{k}^{-1} \sin \theta_{k}}{\log (R / n)} \leqq L .
$$

Replace $R / n$ by $R$ in (6). Finally since $n$ can be taken arbitrarily large, we have 


$$
\limsup _{R \rightarrow \infty} C(R) \leqq L,
$$

from which (4) follows. The proof of the converse is essentially the same and will not be carried out.

We next show that

$$
R^{-2} \int_{1}^{R} \log |f(x) f(-x)| d x=O(1)
$$

Since $f(z)$ is of exponential type, we have at once

$$
R^{-2} \int_{1}^{R} \log |f(x) f(-x)| d x \leqq O(1) .
$$

Now form a function with real zeros which are the moduli of the zeros of $f(z)$, and denote the new function by

$$
F(z)=\prod_{k=1}^{\infty}\left(1-z^{2} / r_{k}^{2}\right)
$$

Then (cf. [1, p. 143]),

(9) $\log \left|\frac{f(x) f(-x)}{F(x)}\right|+\log \left|\frac{f(x) f(-x)}{F(-x)}\right|=2 \sum_{k=1}^{\infty} \log \left|\frac{z_{k}^{2}-x^{2}}{r_{k}^{2}-x^{2}}\right|>0$

since every summand on the right-hand side is non-negative.

Since $F(z)$ has only real zeros, it follows $[1, \mathrm{pp} .86-88]$ that

$$
R^{-2} \int_{1}^{R} \log |F(x) F(-x)| d x=O(1) .
$$

From (9) and (10) we can deduce (7). The theorem now follows from (2), (3), (4), and (7).

Corollary. If $f(z)$ is of exponential type, and $h(\theta)$ is its indicator function, if $\lim \sup _{R \rightarrow \infty} C(R)=L$, and if $\sum_{r_{k} \leq R} R^{-2} r_{k} \sin \theta_{k}=o(\log R)$, then $h(0)+h(\pi) \geqq 2 \pi L$. In particular, if $f(z)$ is of exponential type and $\lim _{R \rightarrow \infty} C(R)=L$, then $h(0)+h(\pi) \geqq 2 \pi L$.

\section{A condition that the limit of the integral exist.}

THEOREM 2. If $f(z)$ is of exponential type, and if

$$
\lim _{x \rightarrow \infty}^{*} x^{-1} \log |f(x) f(-x)|=K,
$$

where the asterisk indicates that the limit exists if one deletes a set of finite logarithmic measure, then $\lim _{R \rightarrow \infty} C(R)=K / 2 \pi$. 
Proof. If the asterisk were not present in (11), Theorem 2 would follow at once from Theorem 1 . Let $E$ be a set of finite logarithmic measure, and let $E(R)$ denote the intersection of $E$ with the interval $[1, R]$. The theorem will be proved if we can show that

$$
\int_{E(R)} x^{-2} \log |f(x) f(-x)| d x=o(\log R)
$$

Since $f(z)$ is of exponential type we have

$$
\int_{E(R)} x^{-2} \log |f(x) f(-x)| d x \leqq O(1) .
$$

Consider the associated function $F(z)$ defined by (8). It will suffice to show that

$$
\int_{E(R)} x^{-2} \log |F(x)| d x \geqq o(\log R),
$$

for (12) will then follow from (14), (9), and (13).

Let us write

$$
F(x)=P_{1}(x) P_{2}(x) P_{3}(x) P_{4}(x),
$$

where each $P_{i}(x)$ is the product formed from certain zeros of $F(z)$. More precisely, $P_{1}(x)$ is the product of factors of the form $\left(1-x^{2} / r_{n}^{2}\right)$ for zeros $r_{n}<x(1-\eta)$, where $\eta$ is some fixed positive number not exceeding 1. $P_{2}(x)$ is the product formed from zeros $r_{n}$ such that $x(1-\eta) \leqq r_{n}<x(1+\eta) . P_{3}(x)$ is the product formed from zeros $r_{n}$ such that $x(1+\eta) \leqq r_{n}<2 x$, and $P_{4}(x)$ is the product formed from the zeros $r_{n}$ such that $r_{n} \geqq 2 x$.

We next proceed to estimate $\int_{E(R)} x^{-2} \log \left|P_{i}(x)\right| d x$ for $i=1,2,3,4$. Since $F(z)$ is of exponential type, there is some constant $T$ such that $n(r) \leqq r T$ for all $r$. Since the number of zeros of $F(x)$ between $x(1+\eta)$ and $2 x$ cannot exceed $2 x T$, it follows that

$$
\log \left|P_{3}(x)\right| \geqq 2 x T \log \left|1-(1+\eta)^{-2}\right|,
$$

and since $E$ is of finite logarithmic length,

$$
\int_{E(R)} x^{-2} \log \left|P_{3}(x)\right| d x \geqq O(1) .
$$

Exactly the same argument shows that

$$
\int_{E(R)} x^{-2} \log \left|P_{1}(x)\right| d x \geqq O(1) .
$$


If we use the series expansion for $\log \left(1-1 / 2^{2 k}\right)$ and compare with a geometric series we see that if $2^{k} x \leqq r_{n}<2^{k+1} x$,

(18) $\log \left|1-x^{2} / r_{n}^{2}\right| \geqq \log \left(1-1 / 2^{2 k}\right)>-2 / 2^{2 k} \quad$ (for $\left.k \geqq 1\right)$.

Since $n(k x) \leqq T k x$, for a given $x$ we can apply (18) to the $r_{n}$ 's in each interval $\left[2^{k} x, 2^{k+1} x\right)$ and add to obtain

$$
\sum_{r_{n} \geq 2 x} \log \left|1-x^{2} / r_{n}^{2}\right|>-4 T x .
$$

From (19) and the fact that $E$ is of finite logarithmic measure, it follows that

$$
\int_{E(R)} x^{-2} \log \left|P_{4}(x)\right| d x \geqq O(1)
$$

It remains to consider $P_{2}(x)$. For $x \geqq 1$,

Now set

$$
\log \left|1-x^{2} / r_{n}^{2}\right|>\log \left|r_{n}-x\right|-2 \log r_{n}
$$

$$
Q_{1}(x)=\sum_{x(1-\eta) \leq r_{n}<x(1+\eta)} \log \left|r_{n}-x\right|
$$

and

$$
Q_{2}(x)=\sum_{x(1-\eta) \leq r_{n}<x(1+\eta)} 2 \log r_{n}
$$

For $x \geqq 2$, we have

(21) $Q_{2}(x) \leqq 2 T x(1+\eta) \log x(1+\eta)<4 T x \log 2 x \leqq 8 T x \log x$.

A routine computation shows that

$$
\int_{E(R)} x^{-1} \log x d x=o(\log R) .
$$

It now follows from (21) and (22) that

$$
\int_{E(R)} x^{-2} Q_{2}(x) d x=o(\log R) .
$$

Finally, we consider the contribution of $Q_{1}(x)$. It is easily seen that over any interval, $\int_{a}^{b} \log \left|r_{n}-x\right| d x \geqq-2$. Thus 


$$
\int_{2^{k-1}}^{2^{k}} x^{-2} Q_{1}(x) d x \geqq-2\left(2^{-2 k+2}\right) \cdot 2^{k} T(1+\eta) \geqq-4 T / 2^{k-2} .
$$

It then follows that

$$
\int_{2}^{\infty} x^{-2} Q_{1}(x) d x \geqq-8 T
$$

a fortiori,

$$
\int_{E(R)} x^{-2} Q_{1}(x) d x=O(1) .
$$

From (15), (16), (17), (20), (23), and (24) we can deduce (14), which proves the theorem.

4. The relation between $C(R)$ and $n(R)$.

TheOREM 3. If $f(z)$ is of exponential type, and lim $\sup _{R \rightarrow \infty} C(R)=L$, then $\lim \sup _{R \rightarrow \infty} R^{-1} n(R) \geqq L$, where $n(R)$ is the number of zeros of $f(z)$ in the upper half-plane with $\left|z_{k}\right| \leqq R$. If $n^{\alpha}(R)$ represents the number of zeros of $f(z)$ in the sector $S=\left\{z:\left|\frac{1}{2} \pi-\arg z\right|<\alpha\right\}$ where $\alpha<\frac{1}{2} \pi$, and if $\lim \inf _{R \rightarrow \infty} C^{\prime}(R)=L$, where the prime indicates summation only over the zeros in $S$, then $\lim \inf _{R \rightarrow \infty} R^{-1} n^{\alpha}(R) \leqq L \sec \alpha$.

Proof. If we write $\sum_{r_{k} \leq R} r_{k}^{-1}$ as a Stieltjes integral and integrate by parts, we obtain

(25) $\sum_{r_{k} \leq R} r_{k}^{-1} \sin \theta_{k} \leqq \sum_{r_{k} \leq R} r_{k}^{-1}=\int_{0}^{R} t^{-1} d n(t)=R^{-1} n(R)+\int_{0}^{R} t^{-2} n(t) d t$.

If we now assume that $\lim \sup _{R \rightarrow \infty} R^{-1} n(R)<L$, and use this in (25), we easily reach a contradiction. The proof of the second assertion is essentially the same.

Both estimates of Theorem 3 are best possible as is easily seen by considering suitable exponential binomials. For example, if $f(z)$ $=1+\exp (\pi L z)$, then $\lim _{R \rightarrow \infty} C(R)=\lim _{R \rightarrow \infty} R^{-1} n(R)=L$. Similarly, if $f(z)=1+\exp \{2 \pi L(-\cos \beta+i \sin \beta) z\}$, then $\lim _{R \rightarrow \infty} C(R)=L \sin \beta$, and for $\alpha>\beta, \lim _{R \rightarrow \infty} R^{-1} n^{\alpha}(R)=L$. By choosing $\beta$ arbitrarily close to $\alpha$, we see that the second estimate is also the best possible.

\section{REFERENCES}

1. R. P. Boas, Jr., Entire functions, Academic Press, New York, 1954.

2. N. Levinson, Gap and density theorems, Amer. Math. Soc. Collog. Publ. Vol. 26, Amer. Math. Soc., Providence, R. I., 1940.

Pennsylvania State University 